OU-HET 358

hep-th/0009021

\title{
Noncommutative Gauge Dynamics from Brane Configurations with Background $B$ field
}

\author{
Nobuyoshi Ohta用 and Dan Tomino" \\ Department of Physics, Osaka University, Toyonaka, Osaka 560-0043, Japan
}

\begin{abstract}
We study $D=3$ field theories on the D3-branes stretched between two NS5-branes with NS $B$-field background. The theory is a noncommutative gauge theory. The mirror symmetry and S-duality of the theory are discussed. A new feature is that the mirror of the noncommutative gauge theory is not a field theory but an open string decoupled from the closed string. We also consider brane creation phenomena and use the result to discuss the analogue of the Seiberg duality. A noncommutative soliton is interpreted as a D1-brane induced on D3-brane.
\end{abstract}

\footnotetext{
*e-mail address: ohta@phys.sci.osaka-u.ac.jp
}

${ }^{\dagger}$ e-mail address: dan@het.phys.sci.osaka-u.ac.jp 


\section{Introduction}

The last few years have witnessed a remarkable progress in our understanding of nonperturbative properties of supersymmetric Yang-Mills theories (SYM) in various dimensions. Many interesting and exact results have been obtained in refs. [1, 2]. These results were interpreted in terms of the field theory realized on the worldvolume of the brane configurations in type IIB superstring theory [3]-[7]. In particular, the miraculous "mirror symmetry" from the field theory point of view can be simply understood as an $S L(2, \mathbf{Z})$ duality symmetry in type IIB superstring theory.

Four-dimensional theories can be also discussed by using brane configurations in type IIA superstring [8]- [10]. The so-called Seiberg duality [11] can be easily understood [8] in terms of brane exchange and creation phenomena first discussed in ref. [4]. These theories have an interpretation in terms of M-brane configuration [10. For a review of these developments, see ref. [7]. In fact, other BPS brane configurations give various field theories including the above-mentioned ones upon compactifications and T-dualities, and it is possible to classify brane configurations [12, 13].

Recently it has been noticed that noncommutative SYM (NCSYM) has also a similar realization on the worldvolume of the branes if we include background NS $B$-field [14]-17]. This naturally leads us to consider various brane configurations discussed so far in the presence of the background $B$ field. NCSYM in type IIA superstring has been considered in ref. [18]. The purpose of this paper is to discuss $D=3$ theories on brane configuration of Hanany-Witten [4] and its variant.

In what follows we first study the $D=3$ NCSYM on D3-branes stretched between two parallel NS5-branes with NS $B$-field background in type IIB superstring. We show the type IIB $S L(2, \mathbf{Z})$ duality symmetry tells us that the mirror of the NCSYM is not just a field theory but noncommutative open string theory (NCOS) [19, 20]. We also consider brane creation phenomena and use the result to discuss the $D=3$ NCSYM version of the Seiberg duality. Our analysis suggests that the analogue of the Seiberg duality is valid also in $D=3$ NCSYM.

The paper is organized as follows. In sect. 2, we briefly review how noncommutative theory is realized on the worldvolume of the branes in the presence of background NS 
$B$-field. The BPS properties of various brane configurations are discussed in sect. 3 . Properties of $D=3 \mathrm{SYM}$ such as mirror symmetry are discussed in sect. 4. In sect. 5, we show that the brane configuration suggests the validity of the NCSYM version of the Seiberg duality. Sect. 6 is devoted to discussions.

\section{Field theory on D-branes with background NS $B$ - field}

In this section we briefly review how noncommutativity appears in the theories realized on D-branes with NS $B$-field background.

The bosonic part of the open string action is given by

$$
S=-\frac{1}{4 \pi \alpha^{\prime}} \int_{\Sigma}\left(g_{i j} \partial_{a} X^{i} \partial^{a} X^{j}-2 \pi \alpha^{\prime} B_{i j} \epsilon^{a b} \partial_{a} X^{i} \partial_{b} X^{j}\right)
$$

where $\Sigma$ is the string worldsheet. The variation of the action (2.1) leads to the boundary conditions

$$
\begin{aligned}
& g_{i j} \partial_{\sigma} X^{j}+\left.2 \pi \alpha^{\prime} B_{i j} \partial_{t} X^{j}\right|_{\partial \Sigma}=0, \quad \text { for } i, j: \text { worldvolume directions, } \\
& \left.g_{m n} \partial_{t} X^{n}\right|_{\partial \Sigma}=0, \quad \text { for } m, n \text { : otherwise. }
\end{aligned}
$$

Note that the first line reduces to the usual Neumann condition in the absence of the $B$ field. The additional term induces the mixing between the "momentum" and coordinates and gives rise to noncommutativity. The canonical momenta are given by

$$
P_{i}=\frac{1}{2 \pi \alpha^{\prime}}\left(g_{i j} \partial_{t} X^{j}+2 \pi \alpha^{\prime} B_{i j} \partial_{\sigma} X^{j}\right)
$$

Imposing the boundary condition (2.2), the mode expansions for fixed $i$ and $j$ are given as

$$
\begin{aligned}
X^{i}= & x^{i}+2 \alpha^{\prime}\left(g^{i j} p_{j} t-2 \pi \alpha^{\prime} B^{i j} p_{j} \sigma\right) \\
& +\sqrt{2 \alpha^{\prime}} \sum_{n \neq 0} \frac{e^{-i n t}}{n}\left(i g^{i j} \alpha_{j n} \cos n \sigma-2 \pi \alpha^{\prime} B^{i j} \alpha_{j n} \sin n \sigma\right), \\
X^{j}= & x^{j}+2 \alpha^{\prime}\left(g^{i j} p_{i} t+2 \pi \alpha^{\prime} B^{i j} p_{i} \sigma\right) \\
& +\sqrt{2 \alpha^{\prime}} \sum_{n \neq 0} \frac{e^{-i n t}}{n}\left(i g^{i j} \alpha_{i n} \cos n \sigma+2 \pi \alpha^{\prime} B^{i j} \alpha_{i n} \sin n \sigma\right) .
\end{aligned}
$$


An interesting property of the mode expansions is that there is a noncommutativity in the coordinates in (2.4) as indicated in the mixing of the momenta and coordinates, but the momenta given in (2.3) have the expansions

$$
\begin{aligned}
2 \pi \alpha^{\prime} P_{i} & =\left[g^{2}+\left(2 \pi \alpha^{\prime} B\right)^{2}\right]_{i j}\left[2 \alpha^{\prime} p^{j}+\sqrt{2 \alpha^{\prime}} \sum_{n} e^{-i n t} i \alpha_{n}^{j} \cos n \sigma\right], \\
2 \pi \alpha^{\prime} P_{j} & =\left[g^{2}+\left(2 \pi \alpha^{\prime} B\right)^{2}\right]_{i j}\left[2 \alpha^{\prime} p^{i}+\sqrt{2 \alpha^{\prime}} \sum_{n} e^{-i n t} i \alpha_{n}^{i} \cos n \sigma\right],
\end{aligned}
$$

and commute with each other.

Imposing the equal-time commutation relations

$$
\left[X^{i}(\sigma, t), P_{j}\left(\sigma^{\prime}, t\right)\right]=i g^{i}{ }_{j} \delta\left(\sigma-\sigma^{\prime}\right),
$$

and that $\left[X^{i}(\sigma, t), X^{j}\left(\sigma^{\prime}, t\right)\right]$ (almost) vanish, we get

$$
\left[x^{i}, x^{j}\right]=i \theta^{i j}, \quad\left[x^{i}, p^{j}\right]=i G^{i j}, \quad\left[\alpha_{n}^{i}, \alpha_{m}^{j}\right]=n \delta_{n+m, 0} G^{i j}
$$

where $G^{i j}$ and $\theta^{i j}$ are the open string metric and noncommutativity parameter defined by

$$
\begin{aligned}
G^{i j} & =\left(\frac{1}{g+2 \pi \alpha^{\prime} B}\right)_{S}^{i j}, \\
G_{i j} & =g_{i j}-\left(2 \pi \alpha^{\prime}\right)^{2}\left(B g^{-1} B\right)_{i j}, \\
\theta^{i j} & =2 \pi \alpha^{\prime}\left(\frac{1}{g+2 \pi \alpha^{\prime} B}\right)_{A}^{i j},
\end{aligned}
$$

with ()$_{S}$ and ()$_{A}$ denoting the symmetric and antisymmetric parts. One then finds

$$
\left[X^{i}(\sigma, t), X^{j}\left(\sigma^{\prime}, t\right)\right]=\left\{\begin{array}{ccc}
i \theta^{i j} & \text { for } & \sigma=\sigma^{\prime}=0 \\
-i \theta^{i j} & \text { for } & \sigma=\sigma^{\prime}=\pi \\
0 & \text { otherwise. } &
\end{array}\right.
$$

Thus the momenta are commutative whereas the coordinates are not at the boundary.

In terms of the Euclideanized coordinate

$$
z=e^{\tau+i \sigma}, \quad \tau \equiv i t
$$

the mode expansion (2.4) and commutation relations (2.7) give the correlator

$$
\begin{aligned}
\left\langle X^{i}(z) X^{j}\left(z^{\prime}\right)\right\rangle= & -\alpha^{\prime}\left[g^{i j} \log \left|z-z^{\prime}\right|-g^{i j} \log \left|z-\bar{z}^{\prime}\right|\right. \\
& \left.+G^{i j} \log \left|z-\bar{z}^{\prime}\right|^{2}+\frac{1}{2 \pi \alpha^{\prime}} \theta^{i j} \log \frac{z-\bar{z}^{\prime}}{\bar{z}-z^{\prime}}+D^{i j}\right], \\
\left\langle X^{m}(z) X^{n}\left(z^{\prime}\right)\right\rangle= & -\alpha^{\prime}\left[g^{m n} \log \left|z-z^{\prime}\right|-g^{m n} \log \left|z-\bar{z}^{\prime}\right|\right] .
\end{aligned}
$$


where $D^{i j}$ is a constant that may depend on $B$ but not on $z$ and $\bar{z}$. On the boundary at $\sigma=0, \pi$, the correlator reduces to

$$
\left\langle X^{i}(\tau) X^{j}\left(\tau^{\prime}\right)\right\rangle=-\alpha^{\prime} G^{i j} \log \left(\tau-\tau^{\prime}\right)^{2}+\frac{i}{2} \theta^{i j} \epsilon\left(\tau-\tau^{\prime}\right)
$$

which gives rise to the star product in the interactions between open string states given by vertex operators [17].

In the scaling limit

$$
\begin{aligned}
\alpha^{\prime} & \sim \epsilon^{1 / 2} \rightarrow 0, \\
g_{i j} & \sim \epsilon \rightarrow 0,
\end{aligned}
$$

the massive and closed string modes decouple and the effective theory of the remaining massless modes on the Dp-brane is NCSYM theory [17]. Though we have discussed the

effective theory only for the bosonic part, once we identify the unbroken supersymmetry in the brane configuration, it is easy to construct the corresponding NCSYM.

Our next task is to identify the BPS brane configurations and which massless modes are kept in the resulting theory.

\section{BPS brane configurations}

In this section, we analyze BPS brane configurations with $B_{12} \neq 0$. The configurations we have in mind are basically the same as those in ref. [4].

\section{$3.1 \quad$ NS5-D5-D3 system}

We first consider type IIB theory in ten-dimensional flat Minkowski space with trivial background fields. The brane configuration of our interest is composed of D3-branes suspended between two parallel NS5-branes with D5-branes for additional matter hypermultiplets. In the flat space, an NS5-brane with the worldvolume in the (012345) directions is invariant under half of the supersymmetries determined by

$$
\epsilon_{L}=\Gamma_{012345} \epsilon_{L}, \quad \epsilon_{R}=-\Gamma_{012345} \epsilon_{R}
$$


Note that the spinors $\epsilon_{L}$ and $\epsilon_{R}$ satisfy $\Gamma \epsilon_{L}=\epsilon_{L}, \Gamma \epsilon_{R}=\epsilon_{R}$ for $\Gamma=\Gamma_{012 \cdots 9}$. Here we use the notation $\Gamma_{012 \cdots p}$ for antisymmetrized product of gamma matrices with unit strength.

On the other hand, a D5-brane with the worldvolume in the (012789) directions is invariant under half of the supersymmetries given by the different condition

$$
\epsilon_{L}=\Gamma_{012789} \epsilon_{R}
$$

Each of these conditions (3.1) and (3.2) preserves 16 components of supersymmetry generators. If there are both the NS5- and D5-branes in flat space, only the supersymmetry compatible with the above two conditions remains unbroken. We thus find that only eight components of the supersymmetry generators are preserved, giving a 1/4 BPS state.

It is possible to add another D3-brane with the worldvolume (0126) to this system without breaking supersymmetry completely. The additional condition we get is

$$
\epsilon_{L}=\Gamma_{0126} \epsilon_{R}
$$

which is automatic given the conditions (3.1) and (3.2) [4].

Next let us introduce a constant NS $B$-field background, $B_{12}=B$ and discuss BPS brane configurations. To examine how the condition (3.3) will be modified by the presence of $B$ field, we go back to the boundary condition (2.2) for $\mathrm{D} p$-brane in the presence of $B$ field. For our D3-brane, this becomes

$$
\begin{aligned}
\partial_{\sigma} X^{0,6} & =0 \\
\partial_{\sigma} X^{1}+2 \pi i \alpha^{\prime} B \partial_{t} X^{2} & =0 \\
\partial_{\sigma} X^{2}-2 \pi i \alpha^{\prime} B \partial_{t} X^{1} & =0 \\
\partial_{t} X^{3,4,5,7,8,9} & =0 .
\end{aligned}
$$

It is then useful to consider the T-dual transformation in the $X^{2}$ direction [18] and note that the T-dual transformation exchanges $\partial_{\sigma}$ with $-i \partial_{t}$. We are thus lead to

$$
\begin{aligned}
\partial_{\sigma} X^{0,6} & =0 \\
\partial_{\sigma}\left(\frac{1}{\sqrt{1+\left(2 \pi \alpha^{\prime} B\right)^{2}}} X^{1}-\frac{2 \pi \alpha^{\prime} B}{\sqrt{1+\left(2 \pi \alpha^{\prime} B\right)^{2}}} X^{2}\right) & =0 \\
\partial_{t}\left(\frac{1}{\sqrt{1+\left(2 \pi \alpha^{\prime} B\right)^{2}}} X^{2}+\frac{2 \pi \alpha^{\prime} B}{\sqrt{1+\left(2 \pi \alpha^{\prime} B\right)^{2}}} X^{1}\right) & =0 \\
\partial_{t} X^{3,4,5,7,8,9} & =0 .
\end{aligned}
$$


This is a D2-brane boundary condition rotated in the (12)-plane by the angle given by $\tan \theta_{12}=-2 \pi \alpha^{\prime} B$. For such rotation, the gamma matrix $\Gamma_{1}$ transforms to $e^{\frac{1}{2} \theta_{12} \Gamma_{12}} \Gamma_{1} e^{-\frac{1}{2} \theta_{12} \Gamma_{12}}=$ $e^{\theta_{12} \Gamma_{12}} \Gamma_{1}$. Thus the unbroken supersymmetry of this $\mathrm{D} 2$-brane is given by

$$
\epsilon_{L}=e^{\theta_{12} \Gamma_{12}} \Gamma_{016} \epsilon_{R}
$$

Making T-duality transformation back in the $X^{2}$ direction, we obtain unbroken supersymmetry of D3-brane in the constant background determined by

$$
\epsilon_{L}=e^{\theta_{12} \Gamma_{12}} \Gamma_{0126} \epsilon_{R}
$$

On the other hand, unbroken symmetry of NS5-brane is unaffected. The condition (3.1) comes from the requirement that the supersymmetry transformation on the NS5brane action be zero [21], but this vanishes for constant NS $B$ field and (3.1) does not change [18].

We now have to examine the remaining supersymmetry under (3.1) and (3.7). The easiest way to identify this is to redefine spinors by

$$
\epsilon_{R}^{\prime}=e^{\frac{1}{2} \theta_{12} \Gamma_{12}} \epsilon_{R}, \quad \epsilon_{L}^{\prime}=e^{-\frac{1}{2} \theta_{12} \Gamma_{12}} \epsilon_{L} .
$$

We then find that the conditions (3.1) and (3.7) reduces back to (3.1) and (3.3) without $B$ in terms of $\epsilon_{L, R}^{\prime}$. We thus conclude that eight supersymmetries are still unbroken for NS5- and D3-branes even in the presence of constant B. Combining (3.1) and (3.7), we can show that the D5-branes are also allowed in the configuration without breaking any supersymmetry.

In summary, we have shown that the configuration with NS5-, D5- and D3-branes keeps eight supersymmetries unbroken in constant background fields (as in the absence of $B$ field (4⿴囗十)).

\subsection{D0 - D4 system - instanton}

As a simple application of the above discussions and check of our method, let us consider a BPS D0-D4 system.

A type IIA configuration with D0- and D4(01234)-branes is a 1/4 BPS configuration in flat Minkowski space without $B_{i j}$. Now, we consider D0- and D4-branes in a constant 
background: $g_{i j}=\eta_{i j}, B_{12} \neq 0, B_{34} \neq 0, B_{i j \neq 1234}=0$. Unbroken supersymmetry is given by

$$
\begin{aligned}
\epsilon_{L} & =e^{\theta_{1} \Gamma_{12}+\theta_{2} \Gamma_{34}} \Gamma_{01234} \epsilon_{R}, \\
\epsilon_{L} & =\Gamma_{0} \epsilon_{R},
\end{aligned}
$$

where the angles are given by $\tan \theta_{1}=-2 \pi \alpha^{\prime} B_{12}, \tan \theta_{2}=-2 \pi \alpha^{\prime} B_{34}$. The first (second) line comes from $\mathrm{D} 4(\mathrm{D} 0)$-brane. The compatibility of these condition requires

$$
\begin{aligned}
\epsilon_{L} & =e^{\theta_{1} \Gamma_{12}+\theta_{2} \Gamma_{34}} \Gamma_{01234}\left(\Gamma_{0}\right)^{-1} \epsilon_{L} \\
& =e^{\theta_{1} \Gamma_{12}+\theta_{2} \Gamma_{34}} \Gamma_{1234} \epsilon_{L} .
\end{aligned}
$$

Thus the number of eigenvalues 1 of the matrix $e^{\theta_{1} \Gamma_{12}+\theta_{2} \Gamma_{34}} \Gamma_{1234}$ gives the number of unbroken supersymmetries. Now $\Gamma, \Gamma_{1234}, i \Gamma_{12}$ and $i \Gamma_{34}$ commute with each other and square to the identity and the traces of their products vanish. So we can diagonalize these matrices as

$$
\begin{aligned}
\Gamma & =\mathbf{1}_{16} \otimes \sigma_{3}, \\
\Gamma_{1234} & =\mathbf{1}_{8} \otimes \sigma_{3} \otimes \mathbf{1}_{2}, \\
\Gamma_{12} & =i \mathbf{1}_{4} \otimes \sigma_{3} \otimes \mathbf{1}_{4}, \\
\Gamma_{34} & =-\Gamma_{12} \Gamma_{1234} \\
& =-i \mathbf{1}_{4} \otimes \sigma_{3} \otimes \sigma_{3} \otimes \mathbf{1}_{2},
\end{aligned}
$$

where $\mathbf{1}_{n}$ is an $n \times n$ unit matrix. We then find

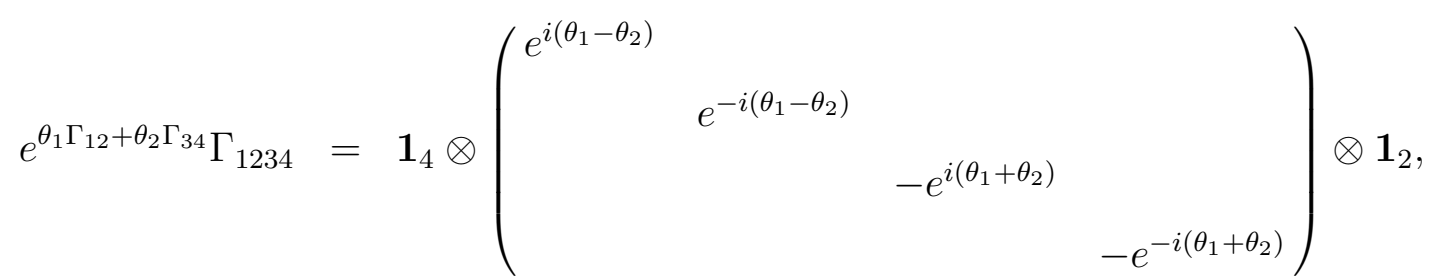

We must take the fact into account that the spinor $\epsilon_{L}$ satisfies the additional condition $\Gamma \epsilon_{L}=\epsilon_{L}$, reducing the number of supersymmetry by half. We then find that in the presence of $B$ field, supersymmetry is unbroken partially if $\theta_{1}=\theta_{2}$ i.e. $B_{12}=B_{34}$ or $\theta_{1}+\theta_{2}=\pi$ i.e. $B_{12}=-B_{34}$, and in both cases eight supersymmetries are preserved. Our result is consistent with that in ref. [17]. 


\section{NCSYM on the branes, brane creation and mirror symmetry}

In this section, we identify the field theory on the worldvolume of the D3-branes in the brane configuration and discuss some nonperturbative properties of NCYM, brane creation and mirror symmetry.

\subsection{Identification of the theory}

It is well known that the effective low-energy theory on the D-branes are SYM theories. From the discussions in sect. 2, it is clear that in the presence of the background NS $B$-field, the theory becomes NCSYM. The supersymmetry on the D3-branes would be $D=3, N=8$, which is reduced by the two parallel NS5-branes to $D=3, N=4$.

Let us first summarize how the massless degrees of freedom on the D3-branes are reduced by the boundary conditions imposed by other branes 4$]$.

1. For D3-branes stretched between two parallel NS5-branes, the massless fields on the D3-branes are the vector multiplets $A_{\mu}, X^{3}, X^{4}, X^{5}$, because Dirichlet conditions are imposed on the directions $X^{6}, \ldots, X^{9}$.

2. For D3-branes stretched between two D5-branes, the massless fields are the hypermultiplets $X^{6}, X^{7}, X^{8}, X^{9}$, because Dirichlet conditions are imposed on the directions $X^{3}, \ldots, X^{5}$.

3. For D3-branes stretched between an NS5-brane and a D5-brane, there remains no massless modes.

As a result, the effective theory on the D3-branes stretched between two parallel NS5branes in the constant NS $B_{12}$ background is a $D=3, N=4$ NCSYM theory with $N=4$ vector multiplets $A_{\mu}, X^{3}, X^{4}, X^{5}$. If we add D5-branes to this system, we get $N=4$ matter hypermultiplets $X^{6}, X^{7}, X^{8}, X^{9}$. This is all the same as [凹], except that the theory is noncommutative in $x^{1}$ and $x^{2}$.

We can interpret geometrical quantities in these configurations as informations on the moduli space of NCSYM on D-branes, just as in ref. [四], at least at the classical level. 
For example, positions of D3-branes are interpreted as the vevs of the scalar fields which parametrize Coulomb and Higgs branches of the $D=3, N=4$ NCSYM, and the length of D3-branes in the $X^{6}$ direction, which is limited by the two NS5-branes, is proportional to the inverse of the gauge coupling constant $1 / g_{Y M}^{2}$ of NCSYM.

There also exist some new aspects such as noncommutative solitons [22], noncommutative monopole [23], and noncommutative instanton [24]. It would be interesting to give a brane realization of these solitonic solutions as in ref. [25]. This will be discussed briefly in sect. 6.

\subsection{Brane creation}

In these brane configurations, matter fields in the fundamental representation in NCSYM come from the open strings stretching between D3-branes and the D5-branes perpendicular to the two NS5-branes. The length of the open strings gives the bare mass to matter fields and this is interpreted as a moduli of NCSYM. The moduli are $X^{3}, X^{4}, X^{5}$ for D5branes. It is important here to realize that $X^{6}$ is irrelevant parameter for NCSYM and the theory is not affected by this position. This observation can be used to argue that brane creation must occur [4] in this system.

The reason is the following. First let us consider $n$ D3-branes between the two NS5branes and denote the positions of the two NS5-branes in the $X^{6}$ direction by $t^{1}$ and $t^{2}$. The D3-brane worldvolume is limited in the $X^{6}$ direction to $t^{1} \leq X^{6} \leq t^{2}$. Now suppose that there are $m$ D5-branes located in the range $t^{1} \leq X^{6} \leq t^{2}$. The low-energy effective theory on the D3-branes in this system is $U(n)$ NCSYM with $m$ hypermultiplets.

Next if we move D5-branes in the $X^{6}$ direction away to the region $X^{6}<t^{1}$ or $t^{2}<X^{6}$, the lengths of the open strings stretching between D3- and D5-branes cannot be zero and hence no massless hypermultiplet does not seem to be produced. However, this cannot be the case since the effective theory does not depend on the position of the D5branes. This leads us to conclude that a new D3-brane must be created between NS5- and D5-branes when the D5-brane crosses through the NS5-brane in the $X^{6}$ direction. The hypermultiplets then arise from the open strings stretching between the new D3-branes and $n$ D3-branes. This process is depicted in Fig. 1. 


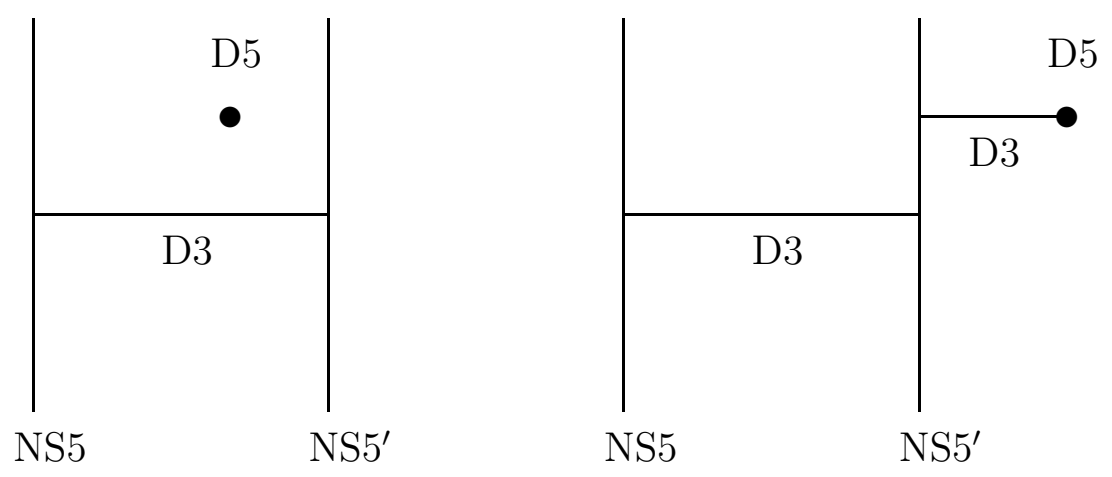

Figure 1: Brane creation

Even in the existence of $B$, the above reasoning does not change. It follows from the same argument that branes must be created. The effect of purely spatial $B$ field is essentially to rescale the momentum and the structure of the string massless modes is not affected (see (2.5)). This is the basic reason why there is no difference in the brane creation even with the $B$ background. For consistency, it is expected that the s-rule is also valid.

Though the consistency of the field theory on the brane requires the brane creation, it would be interesting to confirm this phenomenon by more explicit calculations like those in refs. 26, 27, 28, 29.

\subsection{Mirror symmetry}

It is known that many $D=3, N=4 \mathrm{SYM}$ theories have dual description in terms of those called mirror theories at the IR fixed point [1]. This phenomenon was understood in ref. [4] by a series of operation in the brane system. It consists of an S-dual transformation and a rotation that maps $X^{j}$ to $X^{j+4}$ and $X^{j+4}$ to $-X^{j}$ for $j=3,4,5$, and this amounts to the exchange of the NS5- and D5-branes. Under this operation, geometrical properties of the brane system do not get modified. The moduli spaces of the resulting two effective theories interchanged under this mirror operation have the same structure. This is what is called mirror symmetry in ref. [4].

Now we wish to consider the mirror symmetry for NCSYM. However, the mirror operation involves the S-dual transformation, under which NS $B$ field is mapped to RR 
2-form field $C$. Unfortunately little is known about the effective theory in RR field backgrounds, and it is difficult to identify what is the resulting theory.

It has been pointed out in ref. [20], however, that the NS $B$-field background can be transformed to that of the gauge field strength on the worldvolume and then one can use the Montonen-Olive duality (electromagnetic duality) on the D3-brane for the S-duality. The electromagnetic duality transformation interchange magnetic field $F_{i j \neq 0}$ to electric field $F_{0 i}$. So under this S-duality, the effective theory on the D3-branes in the background $B_{i j \neq 0}$ is mapped into a theory with the background $B_{0 i}$. String theory in the background $B_{0 i}$ is studied in refs. [19, 20]. This theory has space-time noncommutativity $\left[X^{0}, X^{i}\right] \neq 0$, and the decoupling limit of the gravity can be defined for a critical value of the background field $B_{0 i}$, but there is no field theory limit. The theory becomes open string with spacetime noncommutativity, the so-called noncommutative open string (NCOS). Thus under the mirror operation on our brane systems, NCSYM is mapped into NCOS. All this result is also confirmed from the dual supergravity description [30, 31, 32]. Geometrically, we expect that there is no change in the moduli space under the mirror operation.

However, there remains a small subtlety in our interpretation. The resulting NCOS theory by the mirror map contains noncommutativity in the $t, x^{6}$ directions, and the latter corresponds to the direction limited by the 5-branes. This imposes a restriction only on the zero mode sector of the open string modes but not on the non-zero modes. With such restriction we expect that when there is a NCSYM, there exist NCOS which is a mirror to NCSYM in the sense of ref. [1].

To summarize, new and interesting aspects of this mirror symmetry are as follows:

- The mirror of a field theory (which is not a usual one but NCSYM) is not a field theory but NCOS, which is still decoupled from the gravity.

- Pure spatial noncommutativity is mapped into space-time noncommutativity.

- In ref. [四, it was shown that the mirror symmetry exchanges the Coulomb and Higgs branches of SYM. Coulomb branch receives quantum corrections whereas the Higgs branch does not because of nonrenormalization theorems. Moduli space of the Higgs branch is the same as that of the instantons. There does not seem to be much change in the moduli space in noncommutative theory in this regard. 


\section{An analogue of the Seiberg duality}

The brane configuration discussed so far consists of D3-branes stretched between two parallel NS5-branes with additional D5-branes for matter hypermultiplets, and the effective theory is $D=3, N=4$ NCSYM.

The problem we would like to discuss here is another nonperturbative effect in $D=$ $3, N=2$ SYM theory known as Seiberg duality [11]. The corresponding configuration is easy to identify; it is simply given by rotating one of the NS5-branes [8, 9]. The rotated NS5-brane will be referred to as NS5'-brane. The brane configuration is then given by

$$
\text { NS5 : (012345), D3: (0126), D5: (012789), NS5' : (012389). }
$$

It can be checked that the remaining supersymmetry is $D=3, N=2$ in the presence of background $B$ field.

Another important ingredient in the discussion is the brane creation phenomena, which we have already seen that indeed it must occur in the previous section.

Combining these two observations, we can show the NCSYM version of the Seiberg duality; the $D=3, N=2 \mathrm{NCSYM}$ with the gauge group $U\left(N_{c}\right)$ and $N_{f}$ matter hypermultiplets are equivalent to the NCSYM with the gauge group $U\left(N_{f}-N_{c}\right)$ and $N_{f}$ matter hypermultiplets for $N_{f}>N_{c}$. The brane exchange and creation process considered in ref. [8] was made in the direction orthogonal to the $B$-field and obviously it is not modified in the presence of $B$-field. After this process, we find that the resulting effective theory on the D3-brane worldvolume is a $D=3$ NCSYM with the gauge group $U\left(N_{f}-N_{c}\right)$ with $N_{f}$ matter hypermultiplets, just as for the ordinary case [ 8 . This is the desired analogue of the Seiberg duality. Though we believe that the above transformation of brane configuration gives a strong evidence of this duality, it is desirable to check this from the field theoretic point of view.

\section{Discussions}

We have constructed BPS brane configurations corresponding to NCSYM and examined some nonperturbative properties of the theories on the worldvolume. For the theory on 
D3-branes suspended between NS5-branes in type IIB superstring, we find that $D=$ $3, N=4$ NCSYM is realized, and the mirror theory of this theory turns out to be NCOS. It is extremely interesting that the mirror of a field theory is not simply a field theory but an open string.

It is also easy to reduce the number of supersymmetry by rotating one of the NS5branes, as can be understood from the results in refs. [12, 113]. We have used one of these configurations with $1 / 4$ supersymmetry to give a strong evidence of the $D=3$ NCSYM version of the Seiberg duality. After a series of brane exchange and creation, we find that the NCSYM theory on the D3-brane worldvolume with the gauge group $U\left(N_{c}\right)$ with $N_{f}$ matter hypermultiplets is equivalent to a NCSYM with the gauge group $U\left(N_{f}-N_{c}\right)$ with $N_{f}$ matter, just as for the ordinary case [8]. Though we believe that the transformation of brane configuration is a strong evidence of this duality, it is desirable to check this from the field theoretic point of view.

This type of the brane configuration is usually considered for T-dualized type IIA configuration of D4 between two NS5's [8], and gives the usual Seiberg duality. However, in noncommutative case, there is a problem. It has been pointed out that the $U(1)$ sector is frozen for the configuration of $N$ D4-branes and the gauge group is $S U(N)$ but not $U(N)$ [10]. However it is believed that a noncommutative $S U(N)$ SYM theory may not be consistent [33]. This problem is currently under study.

The above discussions look similar to the ordinary SYM. However, from the field theory viewpoint, the properties of the NCSYM under mirror symmetry and Seiberg duality are quite nontrivial. Only with the brane technique and our identification of the suitable BPS brane configuration is it possible to cast this difficult problem to a tractable one. It is then important to understand clearly to what extent the argument remains the same and where it is modified. Our results on the mirror symmetry and Seiberg duality were not known and are already nontrivial for noncommutative SYM. In addition, there are several crucial new features. First, as emphasized above, the mirror theory of the NCSYM is not just a field theory but an open string with space-time noncommutativity decoupled from the closed string.

Another interesting difference is the existence of the nontrivial soliton states in NC theories [22]-[24]. It would be interesting to try to identify such solutions in our brane 
configurations [25]. In this connection, it has been suggested that the soliton in noncommutative scalar field theory [22] can be interpreted as a D-brane [34]. Similar solitonic solutions with constant field strength are considered for NCSYM in ref. [22], and we suspect that these can be again interpreted as D-branes in the NCSYM on the brane configurations.

The Chern-Simons coupling in the effective theory on the $\mathrm{D} p$-branes

$$
\mu_{\mathrm{D} p} \int_{\mathrm{D} p} C \wedge 2 \pi \alpha^{\prime} B=N_{\mathrm{D}(p-2)} \mu_{\mathrm{D}(p-2)} \int_{\mathrm{D}(p-2)} C
$$

produces $\mathrm{D}(p-2)$-brane charge $N_{\mathrm{D}(p-2)}$ for nonzero NS $B$-field background. This means that $N_{\mathrm{D}(p-2)} \mathrm{D}(p-2)$-branes are induced on the $\mathrm{D} p$-brane. In our $D=3$ theory, using $\mu_{\mathrm{D} p}=\frac{\pi}{\kappa^{2}}\left(4 \pi^{2} \alpha^{\prime}\right)^{3-p}$, one finds

$$
N_{\mathrm{D} 1}=\frac{1}{2 \pi} \int B
$$

The effective D-brane action for noncommutative SYM is given by

$$
L=-\frac{1}{G_{s}(2 \pi)^{3} \alpha^{\prime 2}} \sqrt{-\operatorname{det}\left(G+2 \pi \alpha^{\prime} \hat{F}\right)},
$$

where $G_{s}$ is the open string coupling defined by [17]

$$
G_{s}=g_{s}\left(\frac{\operatorname{det}\left(g+2 \pi \alpha^{\prime} B\right)}{\operatorname{det} g}\right)^{1 / 2}
$$

Expanding the action, we find

$$
L=-\frac{\sqrt{-g_{00} g_{33}}}{(2 \pi)^{2} g_{s}^{\prime} \alpha^{\prime 2}}|B|-\frac{1}{2} \frac{\sqrt{-g_{00} g_{33}}}{(2 \pi)^{4} g_{s}^{\prime}} \frac{k_{1}}{|B|}-\frac{k_{2}}{4 g_{Y M}^{2}} \hat{F}_{G}^{2}+O\left(\alpha^{\prime}\right),
$$

where $g_{Y M}^{2}=2 \pi g_{s}, g_{s}=\alpha^{\prime} g_{s}^{\prime}$ and we have assumed a diagonal metric $g=\operatorname{diag} .\left(g_{00}, g_{11}, g_{22}, g_{33}\right)$ and have written $g_{11}=\alpha^{\prime 2} k_{1}, g_{22}=\alpha^{\prime 2} k_{2}$. The first term is of order $\alpha^{\prime-2}$ and gives an infinite contribution in the $\alpha^{\prime} \rightarrow 0$ limit. This term is equal to $\mu_{D 1} N_{D 1} \sqrt{-g_{00} g_{33}}$. This is the contribution of D1-branes with worldvolume in the (03) directions. Thus the introduction of $B$ field indeed gives the correct D1-brane tension. Since a nonvanishing field strength is equivalent to introducing $B$ field, this suggests that the above solution with constant field strength can be interpreted as a D1-brane.

The second term is finite in the $\alpha^{\prime} \rightarrow 0$ limit. This term gives a contribution of the background charge to the low-energy $\left(\alpha^{\prime} \rightarrow 0\right)$ field theory. It can be written as 
$\frac{1}{4} \frac{\sqrt{-\operatorname{det} G}}{g_{Y M}^{2}}\left(\theta^{-1}\right)_{G}^{2}$. The third term is the NCSYM Lagrangian. In these expressions, the suffix $G$ indicates that the contraction of the Lorentz indices are made by the open string metric (2.8). These terms can be combined into

$$
-\frac{\sqrt{-\operatorname{det} G}}{4 g_{Y M}^{2}}\left(\hat{F}-\theta^{-1}\right)_{G}^{2},
$$

which gives the effective action of the NCSYM on the brane.

\section{Acknowledgements}

One of us (N.O.) would like to thank Paul K. Townsend for valuable discussions at SI2000. Preliminary results were reported at IIAS (Kyoto) in June and also at APCTP-Yonsei summer workshop (Seoul) in August. He thanks B.-H. Lee, K. Lee and S. Terashima for useful discussions on the latter occasion, and the organizers for hospitality.

This work was supported in part by Grants-in-Aid for Scientific Research Nos. 99020, 12640270 and in part by Grant-in-Aid on the Priority Area: Supersymmetry and Unified Theory of Elementary Particles.

\section{References}

[1] K. Intriligator and N. Seiberg, Phys. Lett. B387 (1996) 513, hep-th/9607207.

[2] J. de Boer, K. Hori and H. Ooguri, Nucl. Phys. B493 (1997) 101, hep-th/9611063.

[3] M. Porrati and A. Zaffaroni, Nucl. Phys. B490 (1997) 107, hep-th/9611201.

[4] A. Hanany and E. Witten, Nucl. Phys. B492 (1997) 152, hep-th/9611230.

[5] J. de Boer, K. Hori, H. Ooguri, Y. Oz and Z. Yin, Nucl. Phys. B493 (1997) 148, hep-th/9612131; J. de Boer, K. Hori, Y. Oz and Z. Yin, Nucl. Phys. B502 (1997) 107, hep-th/9702154.

[6] S. Elitzur, A. Giveon, D. Kutasov, E. Rabinovici and A. Schwimmer, Nucl. Phys. B505 (1997) 202, hep-th/9704104. 
[7] A. Giveon and D. Kutasov, Rev. Mod. Phys. 71 (1999) 983, hep-th/9802067.

[8] S. Elitzur, A. Giveon and D. Kutasov, Phys. Lett. B400 (1997) 269, hep-th/9702014.

[9] J.L.F. Barbon, Phys. Lett. B402 (1997) 59, hep-th/9703051.

[10] E. Witten, Nucl. Phys. B500 (1997) 3, hep-th/9703166.

[11] N. Seiberg, Nucl. Phys. B435 (1995) 129, hep-th/9411149.

[12] N. Ohta and P.K. Townsend, Phys. Lett. B418 (1998) 77, hep-th/9710129.

[13] T. Kitao, K. Ohta and N. Ohta, Nucl. Phys. B539 (1999) 79, hep-th/9808111; T. Kitao and N. Ohta, Nucl. Phys. B578 (2000) 215, hep-th/9908006.

[14] M. Douglas and C. Hull, J. High Energy Phys. 02 (1998) 008, hep-th/9711165.

[15] F. Ardalan, H. Arfaei and M.M. Sheikh-Jabbari, J. High Energy Phys. 02 (1999) 016, hep-th/9810072.

[16] C.-S. Chu and P.-M. Ho, Nucl. Phys. B550 (1999) 151, hep-th/9812219.

[17] N. Seiberg and E. Witten, J. High Energy Phys. 09 (1999) 032, hep-th/9908142.

[18] M.M. Sheikh-Jabbari, hep-th/0001089.

[19] N. Seiberg, L. Susskind and N. Toumbas, hep-th/0005015.

[20] R. Gopakumar, J. Maldacena, S. Minwalla and A. Strominger, hep-th/0005048.

[21] E. Bergshoeff, R. Kallosh, T. Ortin and G. Papadopoulos, Nucl. Phys. B502 (1997) 149, hep-th/9705040.

[22] R. Gopakumar, S. Minwalla and A. Strominger, hep-th/0003160.

[23] A. Hashimoto and N. Itzhaki, Phys. Lett. B465 (1999) 142, hep-th/9907166; A. Hashimoto and K. Hashimoto, J. High Energy Phys. 11 (1999) 005; D. Gross and N. Nekrasov, hep-th/00052186.

[24] N. Nekrasov and A. Schwarz, Comm. Math. Phys. 198 (1998) 689, hep-th/9802068. 
[25] B.-H. Lee, H.-j. Lee, N. Ohta and H.S. Yang, Phys. Rev. D60 (1999) 106003, hepth/9904181.

[26] C. Bachas, M. Douglas and M. Green, J. High Energy Phys. 07 (1997) 002, hepth/9705074.

[27] U. Danielsson, G. Ferretti and I.R. Klebanov, Phys. Rev. Lett. 79 (1997) 1984, hepth/9705084.

[28] O. Bergman, M. Gaberdiel and G. Lifschytz, Nucl. Phys. B509 (1998) 194, hepth/9705130.

[29] T. Kitao, N. Ohta and J.-G. Zhou, Phys. Lett. B428 (1998) 68, hep-th/9801135.

[30] J.G. Russo and M.M. Sheikh-Jabbari, hep-th/0006202.

[31] R.-G. Cai and N. Ohta, Prog. Theor. Phys. 104 (2000) 1073, hep-th/0007106.

[32] J.X. Lu, S. Roy and H. Singh, hep-th/0007168.

[33] A. Armoni, hep-th/0005208.

[34] J.A. Harvey, P. Kraus, F. Larsen and E.J. Martinec, hep-th/0005013. 